\title{
Nonmodal nonlinear route of transition to two-dimensional turbulence
}

\author{
Aditi Sengupta $\odot,{ }^{1, *}$ Prasannabalaji Sundaram $\odot, 2, \dagger$ and Tapan K. Sengupta $\odot^{2, \$}$ \\ ${ }^{1}$ Department of Engineering, University of Cambridge, Cambridge CB2 1PZ, United Kingdom \\ ${ }^{2}$ High Performance Computing Laboratory, Indian Institute of Technology Kanpur, Kanpur-208 016, India
}

(Received 23 September 2019; accepted 8 January 2020; published 6 February 2020)

\begin{abstract}
Turbulence has remained an unsolved problem in physics, despite the availability of some numerical results. The onset and growth of disturbances leading to two- and three-dimensional turbulence have been theoretically and computationally shown for wall excitation with the response having modal and nonmodal components in the spectrum. The nonmodal component is seen to be dominant. Here, we conclusively show the nonmodal growth for a transition to turbulence, with the same equilibrium flow excited from the free stream with results from linearized and nonlinear two-dimensional Navier-Stokes equations. We establish that transition to turbulence definitely requires nonlinearity, even if the onset process is similar to a linear mechanism. Thus, the transition to turbulence in wall-bounded flows is due to a nonmodal nonlinear mechanism for any disturbance.
\end{abstract}

DOI: 10.1103/PhysRevResearch.2.012033

The absence of a complete theory limits our understanding of turbulence. The accepted formulation to describe turbulent flows is still the Navier-Stokes equation (NSE) [1,2]. While the nonlinearity of the NSE prevents an analytical solution, there are linear theories [3-5] for the onset process. This is the modal approach which studies each normal eigenmode in isolation, and has been the standard approach after the disturbance field included viscous terms for flow over a zero pressure gradient boundary layer. This is known as the OrrSommerfeld equation (OSE), first solved by Heisenberg [6] using approximate analytical tools.

The same approach was followed $[7,8]$ in formulating disturbance growth in space, for a fixed frequency excitation. The authors reported the existence of a Tollmien-Schlichting (TS) wave following this spatial theory, the experimental verification of which came after a careful design of experiments [9] for two-dimensional (2D) transition. The actual role of the TS wave in causing transition has not been shown, which led to a critique of this theory that it tells nothing about turbulence, or about the details of its initial appearance, but it does explain why the original laminar flow can no longer exist [10].

The OSE makes a parallel flow approximation and hence provides local information only. A fundamental problem of the spatial theory lies in assuming the response to be at the excitation frequency. Thus, when the spatiotemporal growth of disturbances was noted by solving the OSE as a receptivity

\footnotetext{
*as2529@cam.ac.uk

†prasanna@iitk.ac.in

¥tksen@iitk.ac.in
}

Published by the American Physical Society under the terms of the Creative Commons Attribution 4.0 International license. Further distribution of this work must maintain attribution to the author(s) and the published article's title, journal citation, and DOI. problem (not as an eigenvalue problem), the response field revealed a three-component structure [11]: a local solution near the exciter, followed by the TS wave, and ahead of these a leading wave front which grows spatiotemporally [11]. This wave packet is termed as a spatiotemporal wave front (STWF), proposed as the forerunner [12] for studies of electromagnetic wave propagation. The presence of STWF justifies studies of hydrodynamic instability without eigenvalues [13] or transition being attributed to nonorthogonal eigenvectors, causing transient growth of energy [14], in the linear framework. The justification for using a Fourier transform in deriving the OSE is provided in linear theory by the Abel and Tauber theorem [15]. These theorems state that one can obtain the (i) near- and far-field response and (ii) the transient and asymptotic response to imposed perturbation by considering different parts of the spectrum. The near field for the fixed frequency excitation is obtained by the essential singularity for the wave number, $\alpha \rightarrow \infty$.

The speed of STWF is given by the group velocity $[16,17]$ of the nonmodal part of the spectrum of the disturbance field. Researchers $[18,19]$ have shown the existence of STWF from the solution of 2D NSE and its role in the creation of 2D turbulence $[20,21]$ with the typical dependence of the energy spectrum on the wave number as $E(\alpha) \sim \alpha^{-3}$. These computations require high accuracy dispersion relation preserving numerical methods [22], which have been subsequently used to show the three-dimensional (3D) routes of transition $[23,24]$.

Two-dimensional turbulence is solved with a finer resolution and is important in atmospheric data [25]; the energy spectrum depicting zonal and meridional wind follows a $2 \mathrm{D}$ trend and accounts for more than $98 \%$ of the total energy. A small fraction follows the well-known $\alpha^{-5 / 3}$ spectrum of 3D homogeneous turbulence.

The inception and growth of flow instability are conjectured to be governed by nonlinear mechanisms [26]. Linear spatial stability theory pronounces pipe and Couette flows to be unconditionally stable for all Reynolds numbers, and 


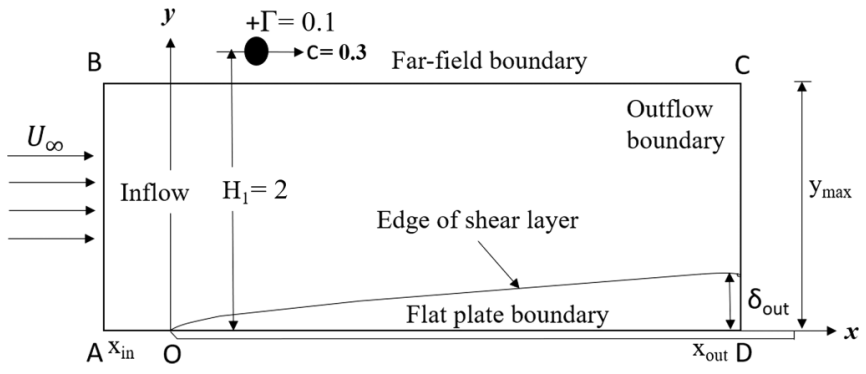

FIG. 1. The computational domain for the receptivity created by a convecting vortex in the free stream over a zero pressure gradient boundary layer on a flat plate.

this discrepancy has prompted researchers [26-28] to seek nonlinear theories as a dynamical system for the evolution from a laminar to turbulent state.

All theoretical and computational efforts discussed so far are for wall-excitation cases. As disturbances are inherently present with incoming free-stream turbulence, research was performed [29] to estimate the critical Reynolds number. A similar physical problem has been studied here as depicted in Fig. 1, for a vortex convecting over the boundary layer. This vortex induces an adverse pressure gradient on the boundary layer, to create recirculating flow packets moving downstream. This unsteady separation is an essential element of the transition. Events are noted in experiments involving turbulent flow over bodies, proposed as a viable transition route [30] created by pressure fluctuations in the oncoming flow. The equilibrium flow is obtained by solving the NSE without any heat transfer, in the absence of the free-stream convecting vortex.

The transition caused by free-stream excitation affects the equilibrium flow globally at each instant of time, causing a time varying adverse pressure gradient which creates unsteady separations. Additionally, the free-stream excitation has an indirect coupling to create an equivalent wall excitation which cannot be easily formulated using OSE. For this reason, in Ref. [31] a linearized NSE was solved, instead of OSE, for the free-stream excitation problem. However, an alternative for solving the full NSE exists, and one studies the flow following a global stability analysis [32]. The problem shown in Fig. 1 has been experimentally [33] and numerically [34] studied. Here, a complete description of the 2D instability and transition to turbulence is provided by a global analysis in the linear and nonlinear frameworks. This will bridge the gap existing for flow transition over a flat plate excited in the free stream. Reporting linear and nonlinear results using an identical numerical framework helps us understand the role played by nonlinearity from the onset to fully developed turbulence. Here, aspects of the linear and nonlinear routes are noted from the spatiotemporal response field over the whole domain to account for both modal and nonmodal contributions. It has been shown in Ref. [35] that transition can occur without the modal component. In the same way, here we show transition without TS waves for free-stream excitation by highly accurate computing, treating this as a global receptivity problem for an aperiodic vortex. In Ref. [36], the authors explain how an imposed 2D disturbance field leads to a strong receptivity mechanism for a vortex of convecting speed $0.3 U_{\infty}$. Using this input, here, we report very high accuracy 2D simulations to emphasize the role of nonlinear mechanism as compared to the linear mechanism.

The linearized and nonlinear NSE are solved for the domain in Fig. 1, using a stream-function-vorticity formulation, which involves solving the vorticity transport and streamfunction equations. Solutions for the disturbance vorticity evaluated from the linearized and nonlinear NSE are obtained by subtracting the equilibrium flow quantities from the instantaneous solution. If the instantaneous velocity and vorticity are given as $\vec{V}$ and $\omega$ for the 2D NSE, then one can write these in terms of equilibrium quantities $\vec{V}_{b}$ and $\omega_{b}$. If the disturbance velocity and vorticity are $\vec{v}_{d}$ and $\omega_{d}$, then the nonlinear NSE (NNSE) is given by the stream-function and vorticity transport equations,

$$
\nabla^{2} \psi=-\omega \text { and } \frac{\partial \omega}{\partial t}+(\vec{V} \cdot \nabla) \omega=\operatorname{Re}^{-1} \nabla^{2} \omega,
$$

where $\psi$ is the instantaneous stream function and the Reynolds number $(\operatorname{Re})$ is defined with the length $(L)$ and velocity scales $\left(U_{\infty}\right)$ and all flow quantities and time instants are nondimensionalized based on $L$ and $U_{\infty}$. Here, $L$ is an arbitrary length measured from the leading edge of the plate where the streamwise Reynolds number based on it is $\mathrm{Re}=$ $10^{5}$. This is the same scale used in Refs. $[19,23,24,36]$. The linearized NSE (LNSE) is given by

$$
\begin{aligned}
\nabla^{2} \psi_{d} & =-\omega_{d}, \\
\frac{\partial \omega_{d}}{\partial t}+\left(\vec{V}_{b} \cdot \nabla\right) \omega_{d}+\left(\vec{v}_{d} \cdot \nabla\right) \omega_{b} & =\operatorname{Re}^{-1} \nabla^{2} \omega_{d} .
\end{aligned}
$$

The solutions of the LNSE and NNSE are obtained using a nonuniform grid in the $x$ and $y$ directions [22]. The computational domain extends from -0.05 to 120 in the $x$ direction and from 0 to 1.5 in the $y$ direction. The simulations have been performed by including the leading edge of the plate inside the computational domain, allowing the inclusion of the leading edge singularity and significant growth of the boundary layer from the leading edge [34]. The results presented here are different from studies which exclude the leading edge and use the Blasius boundary layer as the equilibrium solution. Here, although the intention is to study the zero pressure gradient boundary layer, the front stagnation point is at the leading edge, thus there is a finite variable pressure gradient. This necessitated a much larger domain in $y$ for the present study compared to other studies using the Blasius boundary layer as the equilibrium flow.

The optimized upwind compact scheme (OUCS3) [22] is used for spatial discretization and an optimal three-stage Runge-Kutta scheme (ORK3) [22] is used for time integration with a step of $\Delta t=2.5 \times 10^{-5}$. The feature of these spatial discretization and time integration schemes is the dispersion relation preserving property [22-24] for a high accuracy solution to capture the onset and propagation of the STWF. Here, the group velocity [12] of the STWF is an important parameter, as it transports energy in the system for all scales.

The problem consists of a free-stream vortex with a convection speed $(c=0.3)$ that displayed strong receptivity in experiments [33]. The evolution of the disturbance vorticity field due to imposed free-stream excitation is shown in Supplemental Video 1 [37], over the full domain using NNSE. 

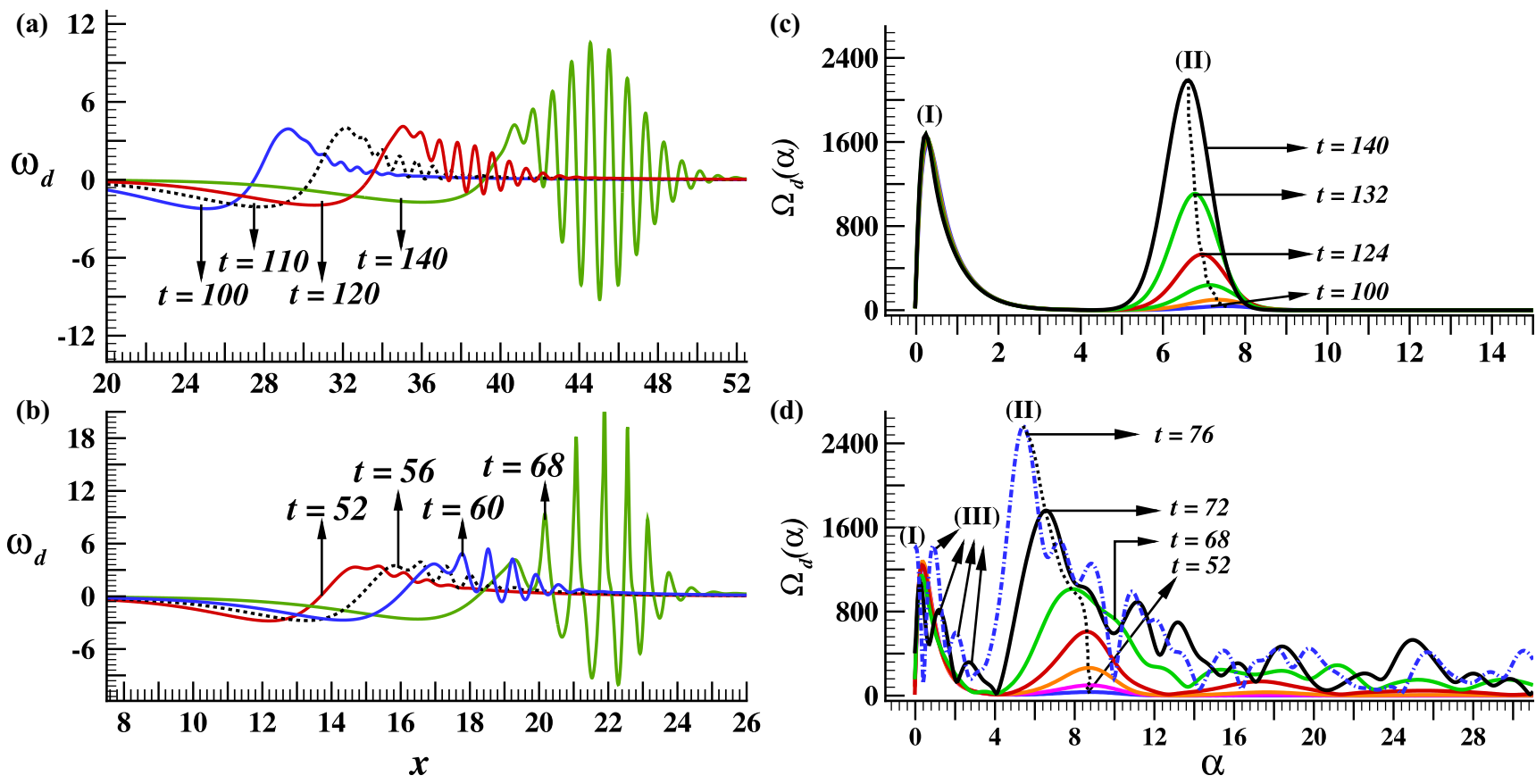

FIG. 2. Disturbance vorticity $\left(\omega_{d}\right)$ and its spectrum at indicated times for $y=0.0058$, evaluated from (a), (c) LNSE and (b), (d) NNSE. While the LNSE indicates a later onset of STWF, the NNSE displays strong effects of nonlinearity in a shorter span of time, as noted at $t=68$. In (d), the spectrum shows the interaction between the local solution and the STWF.

The equilibrium solution computed without any excitation is used for the receptivity calculations, with the free-stream vortex released from $x=-1, y=2$ at $t=0$. Here, $\operatorname{Re}=10^{5}$ and the Reynolds number based on the displacement thickness at the outflow is 5960 .

The onset and propagation of the STWF are noted from the solutions of LNSE and NNSE in Figs. 2(a) and 2(b), respectively. For the LNSE, the early indication of STWF is noted at $t=100$, which emerges out of the local solution, always below the translating vortex. Subsequently, the STWF grows exponentially in space and time, as given by the linear theory. For LNSE, by $t=140$, the peak-to-peak amplitude of the STWF overtakes the amplitude of the local solution. The local solution for LNSE remains invariant. Comparatively, in Fig. 2(b) for NNSE, the onset of the STWF is earlier at $t=52$. Furthermore, the local solution Fourier amplitude decreases with time for NNSE, and by $t=68$, additional peaks appear in its vicinity due to interactions with the STWF. The growth of the STWF given by NNSE indicates an identical onset mechanism as given by LNSE. However, the nonlinear effects are significantly different from the linear mechanism. The formation of STWF is by the same mechanism, as in the wall-excitation case $[11,19,23,24]$. In Fig. 2(b), at $t=68$, nonlinear growth of the STWF is observed. Along with an earlier onset, the saturation of the amplitude for the STWF is also rapid for NNSE, while LNSE shows a later onset and no saturation. The nonlinear saturation for NNSE indicates multiple length scales in Fig. 2(d), while the LNSE shows the same length scale, as noted in Fig. 2(c). Thus, to correctly understand the dynamics during transition, one must use the NNSE.
The local solution is indicated by (I) in Figs. 2(c) and 2(d), and the STWF is identified by (II). The STWF is due to constructive interference of the nonmodal part of the spectrum. The maximum wave number of the peak is traced by a dotted line, which identifies the STWF. In Fig. 2(d) at $t=60$, in addition to the peak for the central wave number, secondary peaks are noted for higher $\alpha$. These additional peaks attain a significant amplitude with time. The bandwidth of the spectra for NNSE is larger. From $t=72$ onwards, there are additional peaks in the spectrum near the local solution component due to interactions with the STWF, marked as (III) in Fig. 2(d). This is in accordance with the Abel's theorem [15], which states that the far field in the physical plane corresponds to the vicinity of origin in the spectral plane. The evolution of the spectra obtained from LNSE and NNSE is shown for $0 \leqslant t \leqslant 165$ in Supplemental Video 2 [37], to show the effects of nonlinearity.

In Fig. 3, the wall-normal distributions of $\omega_{d}$ from the solution of LNSE and NNSE are plotted at $t=120, x=$ 38.7 and $t=60, x=18.5$, respectively. These times represent the linear evolution stage of the STWF for both LNSE and NNSE and the STWFs are centered around the chosen spatial locations. The $\omega_{d}$ decays with height and an inner maximum is noted near $y=0.0058$. The height of the inner maximum is a weak function of time. Thus, the results presented here are shown for this height.

The nonlinear saturated state of the STWF and its spectrum are shown in Fig. 4. Such wideband spectra of the signal shown in Figs. 4(a)-4(c) have also been noted for the wallexcitation case [19]. The discrete times in Fig. 4 have been specifically chosen to show the formation of intermittent 


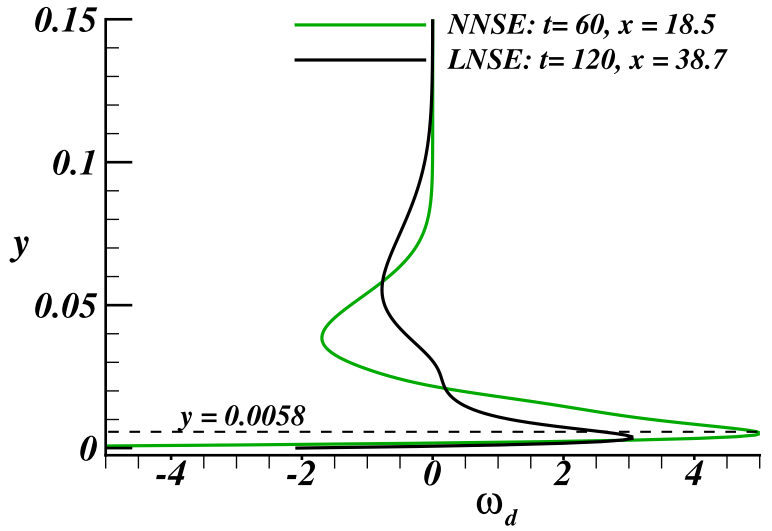

FIG. 3. Wall-normal distribution of disturbance vorticity from the solution of LNSE (at $t=120, x=38.7$ ) and NNSE (at $t=60$, $x=18.5$ ) showing an inner maximum near $y=0.0058$.

spikelike structures in the physical plane with the formation of a vortex doublet, as shown in Fig. 4(b) at $x_{o}=38.75$. This is akin to a singularity added at that time, which is localized in space and causes the spectrum in Fig. 4(e) with a qualitative difference. By $t=99$, the vortex doublet disappears, and the spectrum in Fig. 4(f) reverts to that observed in Fig. 4(d). The sudden appearance of a spike adds a band-limited component in the form of $e^{i \alpha x_{o}}$ at all wave numbers (which can be related by an equivalent time shift property of the Fourier-Laplace transform [38]). Such nonlinear events altering the wideband signal are a very special effect of nonlinearity for a fluid dynamical system, seen clearly at high wave number ranges of the spectrum in Fig. 4(e). Such events repeatedly occur
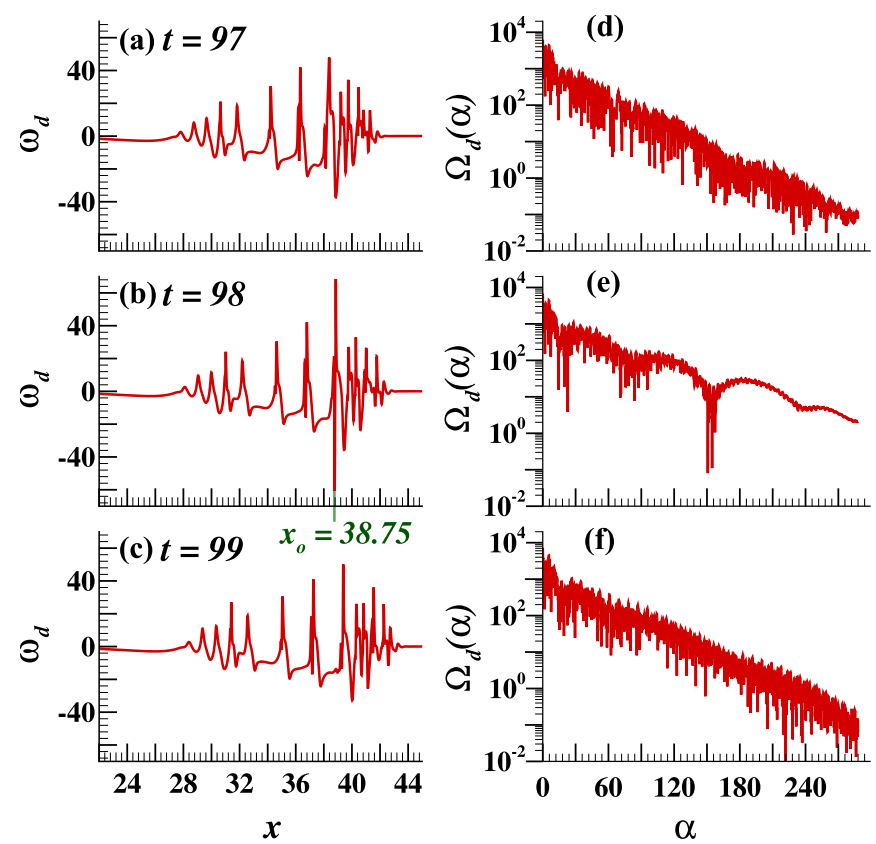

FIG. 4. (a)-(c) Disturbance vorticity as a function of $x$ at indicated times and (d)-(f) the corresponding Fourier transforms $\left[\Omega_{d}(\alpha)\right]$, evaluated from NNSE for $y=0.0058$. Wideband shift in the spectrum in (e) is observed due to the intermittent formation of spikes in the STWF in (b) at $x_{o}=38.75$.

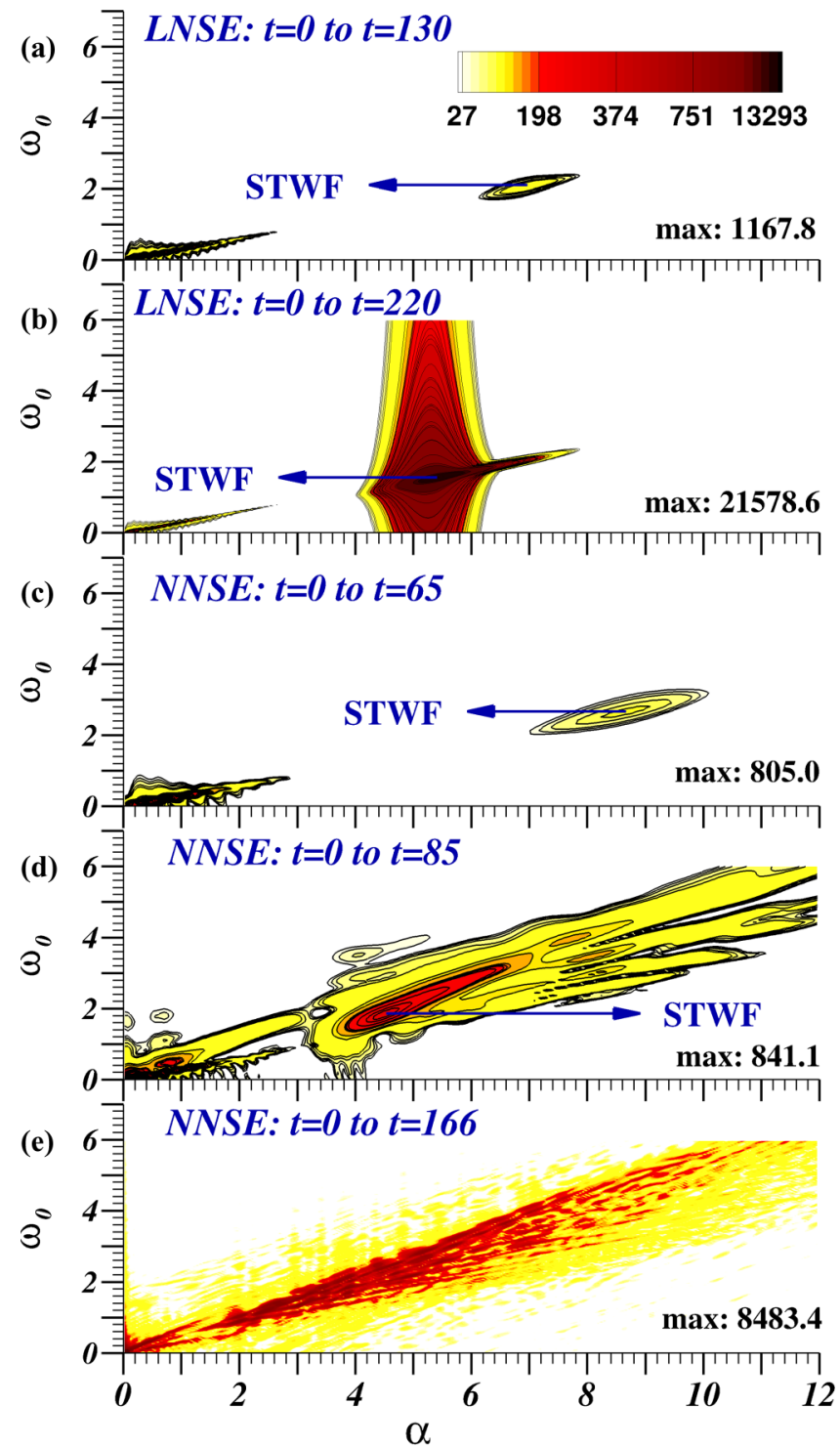

FIG. 5. Fourier-Laplace transform of $\omega_{d}$ for $y=0.0058$ in the $\left(\alpha, \omega_{o}\right)$ plane showing the local solution (near the origin of the spectral plane) and the STWF from (a), (b) LNSE and (c)-(e) NNSE for the indicated time ranges. The evolution of STWF for NNSE transforms the single peak in (c) to a dispersed nonmodal spectrum in (d) and (e).

whenever secondary separation bubbles form on the wall. This has been identified and termed as twinkling of the spectrum. The time-resolved depiction of this phenomenon is shown in Supplemental Video 3 [37]. The attributes of the nonlinearity are explained further in Fig. 5, with the spectra shown in the wave-number-frequency plane for the indicated time ranges.

The spectrum of $\omega_{d}$ obtained from the LNSE and NNSE are shown in Fig. 5. The group velocity of the STWF in the early stage can be calculated from the spectra in Figs. 5(a)-5(d) from $d \omega_{o} / d \alpha$ at the peak amplitude, marked as STWF. For the spectrum in Fig. 5(a), the local solution dominates over the STWF. In Fig. 5(b), the spectrum shows that the local solution and the STWF retain distinct identities, with the local solution retaining its amplitude with time, while the STWF grows exponentially for the LNSE. The wave 


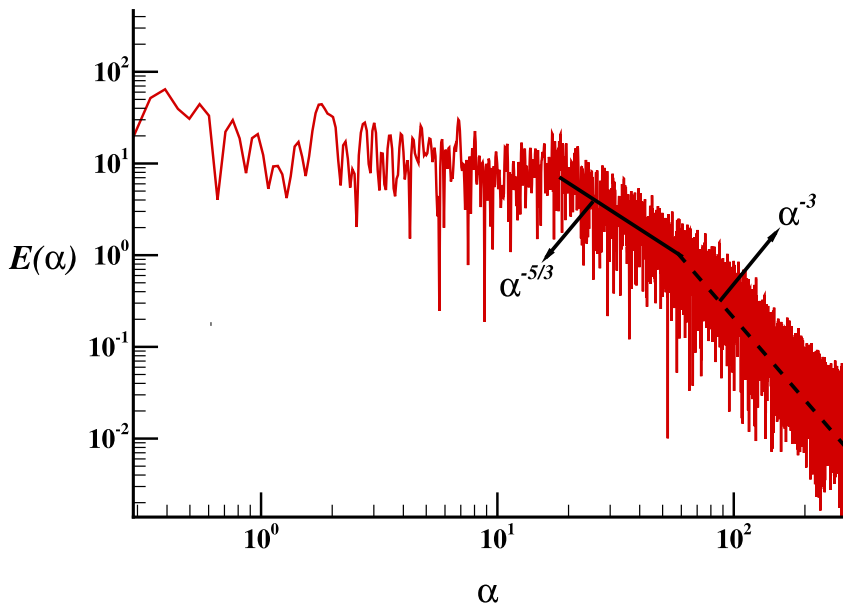

FIG. 6. Energy spectrum of the solution of NNSE at $t=165$ and $y=0.0058$ showing $-5 / 3$ and -3 type variation, typical of $2 \mathrm{D}$ turbulence [2].

number and frequency of the STWF change slowly with time for the LNSE. For the NNSE in Fig. 5(c), one notes the onset of STWF with the effects of nonlinearity accelerating the growth significantly. For the spectrum in Figs. 5(d) and 5(e), the effects of nonlinearity cause a significant dispersion over a wide range of wave numbers and frequencies. The spectrum of the local solution is also affected by the appearance of a higher amplitude and additional peaks induced by the nonlinear interaction.

Usually, 2D turbulence is characterized by a direct cascade (dictated by the enstrophy) for which $E(\alpha) \sim \alpha^{-3}$ [2]. This has been shown in Fig. 6. Also, in 2D turbulence an inverse cascade exists, given as $E(\alpha) \sim \alpha^{-5 / 3}$, which has also been marked in Fig. 6. In Fig. 3.8 of Ref. [2], the direct cascade is referred to as an enstrophy cascade while the inverse cascade is referred to as an energy cascade. This is typical of fully developed 2D turbulence [20,21]. This highlights the importance of working with a nonlinear, spatiotemporal framework to account for all stages of the receptivity problem considered for a global, nonmodal nonlinear analysis.

In conclusion, the routes to turbulence for the zero pressure gradient boundary layer excited at the wall have been shown earlier as due to the STWF in Refs. [11,19,23,24]. Recently, it has been shown that the STWF is the definitive causal mechanism for the onset of transition to turbulence, irrespective of the nature of the imposed wall excitation [35]. For the same equilibrium flow in Refs. [29,30], the authors have discussed the receptivity of flow to free-stream turbulence. Such transition is triggered by the induced unsteady pressure gradient on the boundary layer causing unsteady separations, as solved here for the NSE in 2D. Here, we have studied the element of receptivity for this canonical flow excited by a convecting vortex in the free stream, using both linear and nonlinear formulations with identical numerical setups. It is noted that the onset of STWF is captured by both formulations. However, the evolution of STWF is completely different following these two formulations and only the nonlinear formulation captures the transition to turbulence. We have explored the long-term nonlinear dynamics of STWF, and shown that the transition occurs due to its growth. The later stages of transition are dictated by secondary nonlinear effects on the STWF, which lead to unsteady separations, resulting in a broadband spectrum. In Figs. 4 and 5, we have shown the nonlinear phase effects and dispersion relation for free-stream excitation. The initial STWF transforms into a turbulent spot creating additional STWFs, which merge downstream to create the turbulent flow. The free-stream excitation case considered here is distinctly different from the wall-excitation case [35], as the local solution and the STWF continually interact for the former, while for the latter, these two components do not interact after the onset of STWF. As a consequence, the linear and nonlinear solutions are completely different for the free-stream excitation case. Here, we have shown a definitive, deterministic route to $2 \mathrm{D}$ turbulence by NNSE, even though the onset may appear similar to a linear mechanism [11]. We have also explained the role for the onset by a nonmodal component, which requires the vital nonlinear growth of STWF in leading the flow to turbulence $[26,28]$. The extension to a $3 \mathrm{D}$ route is straightforward, with initial results presented in Ref. [36], where a parametric study of speed, strength, and sign of rotation of the vortex is performed. This was done with the aim of corroborating the results with experiments in Ref. [33].

Here, based on the most optimal speed of the vortex (obtained from Ref. [36]), we have carried out highly resolved 2D simulations to distinguish between the linear and nonlinear operators of the NSE in global receptivity to free-stream excitation. Prior studies on wall excitation [11,19,23,24] showed that the response field consists of modal and nonmodal components, but it is the STWF (nonmodal component) which is the precursor to transition. Here, we show that the STWF plays a primary role even for free-stream excitation, for both solutions of LNSE and NNSE. The distinction between the mechanisms followed by the STWF in solutions from LNSE and NNSE are found to be important for the free-stream excitation case. In wall excitation, this distinction is not prominent. While the receptivity to wall excitation is essentially a carefully designed laboratory scenario, the free-stream excitation case is a more realistic practical flow, and thus this kind of detailed global receptivity study is essential. The present work provides a complete picture for wall-bounded turbulence, by distinguishing between wall and free-stream excitations, and then between global linear and nonlinear receptivity.
[1] G. K. Batchelor, An Introduction to Fluid Dynamics (Cambridge University Press, Cambridge, UK, 1988).

[2] C. R. Doering and J. D. Gibbon, Applied Analysis of the NavierStokes Equations (Cambridge University Press, Cambridge, UK, 1995).
[3] W. McF. Orr, The stability or instability of the steady motions of a perfect liquid and of a viscous liquid. Part I: A perfect liquid, Proc. Roy. Irish Acad. A 27, 9 (1907); The stability or instability of the steady motions of a perfect liquid and of a viscous liquid. Part II: A viscous liquid, 9, 69 (1907). 
[4] L. Rayleigh, On the stability, or instability, of certain fluid motions, Proc. London Math. Soc. 11, 57 (1880).

[5] A. Sommerfeld, Ein Beitrag zur hydrodynamischen Erklärung der turbulenten Flüssigkeitsbewegungen, Proceedings of the 4th International Congress of Mathematicians, Rome, Vol. III (Accademia dei Lincei, 1909), pp. 116-124.

[6] W. Heisenberg, Über stabilität und turbulenz von flüssigkeitsströmen, Ann. Phys. (Leipzig, Ger.) 379, 577 (1924).

[7] H. Schlichting, Zur entstehung der turbulenz bei der plattenströmung, Nachr. Ges. Wiss. Goettingen, Math.-Phys. K1. 181 (1933).

[8] W. Tollmien, Über die enstehung der turbulenz, Nacht. Ges. Wiss. Gottingen. Math.-phys. K1. 21 (1931) [Translated as The production of turbulence, Tech. Memor. Nat. Adv. Comm. Aero., Wash. No. 609 (1931)].

[9] G. B. Schubauer and H. K. Skramstad, Laminar boundary layer oscillations and the stability of the laminar flow, J. Aerosol Sci. 14, 69 (1947).

[10] L. M. Mack, Boundary layer stability theory, In AGARD Special Course on Stability and Transition of Laminar Flow, AD A147 243 (1984).

[11] T. K. Sengupta, A. Kameswara Rao, and K. Venkatasubbaiah, Spatio-Temporal Growing Wave Fronts in Spatially Stable Boundary Layers, Phys. Rev. Lett. 96, 224504 (2006).

[12] L. Brillouin, Wave Propagation and Group Velocity (Academic, New York, 1960).

[13] L. N. Trefethen, A. E. Trefethen, S. C. Reddy, and T. A. Driscoll, Hydrodynamic stability without eigenvalues, Science 261, 578 (1993)

[14] P. J. Schmid, Nonmodal stability theory, Annu. Rev. Fluid Mech. 39, 129 (2007).

[15] B. van der Pol and H. Bremmer, Operational Calculus based on Two-Sided Laplace Integral (Cambridge University Press, Cambridge, UK, 1959).

[16] L. Rayleigh, Scientific Papers 1 (Cambridge University Press, Cambridge, UK, 1889).

[17] L. Rayleigh, Scientific Papers 2 (Cambridge University Press, Cambridge, UK, 1890).

[18] T. K. Sengupta, Instabilities of Flow and Transition to Turbulence (CRC Press, Boca Raton, FL, 2012).

[19] T. K. Sengupta and S. Bhaumik, Onset of Turbulence from the Receptivity Stage of Fluid Flows, Phys. Rev. Lett. 107, 154501 (2011).

[20] G. K. Batchelor, Computation of the energy spectrum in homogeneous two-dimensional decaying turbulence, Phys. Fluids 12, 233 (1969).

[21] R. H. Kraichnan and D. Montgomery, Two-dimensional turbulence, Rep. Prog. Phys. 43, 547 (1980).

[22] T. K. Sengupta, High Accuracy Computing Methods: Fluid Flows and Wave Phenomena (Cambridge University Press, New York, 2013).
[23] S. Bhaumik and T. K. Sengupta, Precursor of transition to turbulence: Spatiotemporal wave front, Phys. Rev. E 89, 043018 (2014).

[24] S. Bhaumik and T. K. Sengupta, Impulse response and spatiotemporal wave-packets: The common feature of rogue waves, tsunami and transition to turbulence, Phys. Fluids 29, 124103 (2017).

[25] G. D. Nastrom, K. S. Gage, and W. H. Jasperson, Kinetic energy spectrum of large-scale and mesoscale atmospheric processes, Nature (London) 310, 36 (1984).

[26] R. R. Kerswell, Nonlinear nonmodal stability theory, Annu. Rev. Fluid Mech. 50, 319 (2018).

[27] B. Eckhardt, T. M. Schneider, B. Hof, and J. Westerweel, Turbulence transition in pipe flow, Annu. Rev. Fluid Mech. 39, 447 (2007).

[28] G. Kawahara, M. Uhlmann, and L. van Veen, The significance of simple invariant solutions in turbulent flows, Annu. Rev. Fluid Mech. 44, 203 (2012).

[29] G. I. Taylor, Statistical theory of turbulence V. Effects of turbulence on boundary layer, Proc. R. Soc. London, Ser. A 156 , 307 (1936).

[30] A. S. Monin and A. M. Yaglom, Statistical Fluid Mechanics: Mechanics of Turbulence (MIT Press, Cambridge, MA, 1971).

[31] T. K. Sengupta, M. Chattopadhyay, Z. Y. Wang, and K. S. Yeo, By-pass mechanism of transition to turbulence, J. Fluids Struct. 16, 15 (2002)

[32] V. Theofilis, Global linear instability, Annu. Rev. Fluid Mech. 43, 319 (2011).

[33] T. T. Lim, T. K. Sengupta, and M. Chattopadhyay, A visual study of vortex-induced subcritical instability on a flat plate boundary layer, Exp. Fluids 37, 47 (2004).

[34] T. K. Sengupta, S. De, and S. Sarkar, Vortex-induced instability of an incompressible wall-bounded shear layer, J. Fluid Mech. 493, 277 (2003).

[35] P. Sundaram, T. K. Sengupta, and S. Sengupta, Is TollmienSchlichting wave necessary for transition of zero pressure gradient boundary layer flow? Phys. Fluids 31, 031701 (2019).

[36] A. Sengupta, V. K. Suman, and T. K. Sengupta, Direct numerical simulation of vortex-induced instability for zero pressure gradient boundary layer, Phys. Rev. E 100, 033118 (2019).

[37] See Supplemental Material at http://link.aps.org/supplemental/ 10.1103/PhysRevResearch.2.012033 for the following videos: Supplemental Video 1: Evolution of disturbance vorticity in the computational domain to illustrate the response field generated by a free-stream convecting vortex using nonlinear formulation. Supplemental Video 2: Evolution of spectra obtained from solutions of linearized and nonlinear Navier-Stokes equation to highlight the effects of nonlinearity. Supplemental Video 3: Time-resolved depiction of the twinkling of the spectrum: Effect of unsteady separation.

[38] A. Papoulis, Fourier Integral and its Applications (McGrawHill, New York, 1962). 